\title{
Entropy and the variability of dependence: System of profound knowledge (SPK) and induced entropy copula as a measure of variability for copula density
}

Jose Luis Guerrero-Cusumano, McDonough School of Business, Georgetown University, USA, guerrerj@georgetown.edu

\begin{abstract}
Deming's System of Profound Knowledge (SPK) is a framework for creating, disseminating, and applying knowledge in business. One of its basic principles is the knowledge of variation. SPK is based on a systemic approach in which each part of the system is interconnected. Process control seeks to determine whether a process is in control, meaning whether the distribution of some critical measured variable is stable. Complex systems may vary through time and their variability can be measured by its entropy and its interrelatedness. The entropy copula or interrelatedness is interpreted as a measure of reduction of independence measured by the entropy of independent variables. This paper provides the expression of the entropy copula for multivariate $t$ distribution and introduces the concept of induced entropy copula as a measure of variability for copula densities. A test for uncorrelatedness based on the entropy copula is also developed. A manufacturing example, using multivariate control chart, is analyzed using the entropy copula approach. Tables are provided in the appendix for testing at different significance levels.
\end{abstract}

Keywords: Deming, profound knowledge, bodies of knowledge, entropy copula, multivariate $t$, asymptotic distribution, test for uncorrelatedness.

\section{Introduction}

Deming (1994) said that "best efforts and hard work, not guided by new knowledge, only dig deeper the pit we are already in" (p. 1). Deming $(1986 ; 1994)$ in a series of books developed the concept of the System of Profound Knowledge (SPK), which set forth a framework for creating, disseminating, and applying knowledge (Hensler et al., 2000). SPK (Deming, 1986) consists of four bodies of knowledge:

- Appreciation of the system (knowledge of the system)

- Theory of knowledge

- Psychology (knowledge of psychology)

- Knowledge of Variation

SPK is based on a systemic approach in which each part of the system is interconnected, where a system is an interconnected complex of functionally related components that work together to try 
to accomplish the aim of the system. The system aim is to be optimized throughout time (Deming, 1986).

There are different types of system behaviors, namely, stable equilibrium, explosive instability and bounded instability (Stacey, 2007). Burnes (2004) suggested that bounded instability, in complex systems, is the only order-disorder that has the ability to transform themselves in order to survive. The concept of bounded instability for a business process is defined as a sequence of behaviors in time, in which the system changes between equilibrium and chaos (Delahaye, 2002). In business, the manager tries to maintain its stability via process of control and measuring its chaos. Business system optimization requires control techniques and methodologies in order to minimize chaos and an excessive increase of entropy (Kullback, 1968). In a complex system, this entropy will depend on the number of interrelated variables defining the system and its multivariate characteristics (Edgeman \& Fraley, 2008).

In situations where one observes a vector of $p$ variables, multivariate processes is deployed frequently in practice, as recent applications of Big Data and Data Mining show. In these processes, the concept of variability and risk become fundamental in order to understand the nature of the data. In a multivariate framework, variability could be defined in several ways; however, it is important to begin with a generalized measure of variability in order to have a better idea of the joint behavior of p-variables, especially when p is large. Guerrero (1994) proposed the use of information theory to analyze and control multivariate processes for different business applications such as quality control and finance. This information theoretic approach extended the concepts of the variability given by covariance and correlation matrix to the concepts of entropy and mutual information.

The basic assumption for multivariate process control is multivariate normality, which is completely specified by its expected value and covariance matrix $\Sigma$. This assumption many times cannot be supported by real data. There is, therefore, the need of creating different alternative schemes for the analysis of multivariate data.

A typical situation that occurs in practice is when the data have "fatter tails" than the corresponding normal distribution. This phenomenon not only occurs in quality control situation but also in financial data. In financial data, there is a constant need of checking volatility and interrelationships among stocks and indices. In order to study these phenomena, when considering different indices, measures of total variability and cohesion are necessary. Modeling using the multivariate normal distribution has proven to be inaccurate especially for fat-tailed distributions (see Blatberg \& Gonedes, 1974; Jöhri, 2004).

In general, fat-tailed distribution is defined as a distribution with the following characteristic:

$$
\operatorname{Pr} o b[X>x]: x^{-(1+\alpha)} \text { As } x \rightarrow \infty \text {, with } \alpha>0
$$

This situation has spurned the use of alternative models such as the multivariate $t$ and Cauchy distribution. This paper assumes the underlying distribution of the observation is the multivariate $t$ distribution. The $t$ distribution plays an important role not only in statistics but also in different areas of business and engineering. Dunnett and Sobel (1954) encountered this distribution in the 
context of certain multiple decision problems, Sutradhar and Ali (1986) generalized the multiple linear regression models for the case that the errors follow a multivariate $t$ distribution.

The use of copulas in multivariate modeling was suggested in an early paper by Hoffding (1940) though was not until 1959 (Sklar, 1959) when copulas becomes a feasible way to analyze complex multivariate distribution dependence structures. The need to go beyond simple structure such as the multivariate normal distribution created the need in complex system to analyze not only the correlation structure but also the dependence structure of a multivariate problem (Hardle Edgeman, \& Guerrero-Cusumano, 2009). Nelson (2006) provided an informative compendium of the theoretical and practical uses of copulas. Copulas have been used in different areas such as quality control (Fernández \& Kolev, 2007), petrochemical sector (Díaz-Viera \& Casar-González, 2005), finance (Bouyé, Durrleman, Nikeghbali, Riboulet, \& Roncalli, 2000; Savu \& Trede, 2010), and insurance industry (Embrechts, 2009). Jaworski, Durante, Härdle, and Rychlik (2010) gave an interesting compendium of copulas applications.

This paper consists of three main parts. The first deals with the definition of entropy copula and its expression for central multivariate $t$ distribution. In this research the concept of induced copula variability introduce. The second part will deal with the sampling distribution of the entropy copula for the multivariate $t$. The third part will be an application to car safety industry.

\section{Preliminaries}

\section{Definition}

A p-dimensional copula is a distribution function on $[0,1]^{\mathrm{p}}$ with standard uniform marginal distributions. Namely $\mathrm{C}(\mathrm{u})=\mathrm{C}\left(\mathrm{u}_{1}, \mathrm{u}_{\mathrm{p}}\right)$. The copula density, when it exists, is given by

$c\left(u_{1}, \ldots, u_{p}\right)=\frac{\partial C\left(u_{1}, \ldots, u_{p}\right)}{\partial u_{1} \mathrm{~L} \partial u_{p}}$

Where the copula $C\left(u_{1}, \ldots, u_{p}\right)$ is defined as,

$C\left(u_{1}, \ldots, u_{p}\right)=F\left[F_{1}^{-1}\left(u_{1}\right), \ldots ., F_{p}^{-1}\left(u_{p}\right)\right]$

$\mathrm{F}$ is an absolutely continuous joint density function with strictly increasing, continuous marginal density function $F_{1} \ldots F_{p}$, the copula density could be simply expressed as

$$
c\left(u_{1}, \ldots, u_{p}\right)=\frac{\partial C\left(u_{1}, \ldots, u_{p}\right)}{\partial u_{1} \mathrm{~L} \partial u_{p}}=\frac{\partial F\left[F_{1}^{-1}\left(u_{1}\right), \ldots, F_{p}^{-1}\left(u_{p}\right)\right]}{\partial u_{1} \mathrm{~L} \partial u_{p}}=\frac{f\left[F_{1}^{-1}\left(u_{1}\right), \ldots ., F_{p}^{-1}\left(u_{p}\right)\right]}{f_{1}\left[F_{1}^{-1}\left(u_{1}\right)\right] \mathrm{L} f_{p}\left[F_{p}^{-1}\left(u_{p}\right)\right]}
$$

Where $f$ is the joint density of $F$ and $f_{1}, \ldots, f_{p}$ are the marginal densities and $F_{i}^{-1}$, are the ordinary inverses of the marginal density functions, therefore the copula density is the ratio of the joint density to what it would have been under independence. Different authors considered multivariate copula constructions (Czado, 2010; Lambert, 2007).

\section{The entropy of the copula density distribution}


The entropy of the copula of any distribution, when it exists is given by

$$
H\left[c\left(u_{1}, \ldots, u_{p}\right)\right]=\int_{[0,1]^{p}}-\log \left[c\left(u_{1}, \ldots, u_{p}\right)\right] c\left(u_{1}, \ldots, u_{p}\right) d U
$$

By performing a simple change of variables $u_{i}=F_{i}\left(x_{i}\right), H\left[c\left(u_{1}, \ldots, u_{p}\right)\right]$ can be alternatively written in terms of the original variables $\mathrm{X}$ as:

$$
H\left[c\left(u_{1}, \ldots, u_{p}\right)\right]=\sum_{i=1}^{p} H_{i}\left(x_{i}\right)-H(X)
$$

Where $\mathrm{H}(\mathrm{X})$ is the entropy of the joint original variables, and $\mathrm{H}\left(\mathrm{x}_{\mathrm{i}}\right)$ is the marginal entropy of variables $\mathrm{x}_{\mathrm{i}} . H\left[c\left(u_{1}, \ldots, u_{p}\right)\right]$ represents a measure of the variability or spread of copula density, it can be regarded as a measure of the deviation of the copula density from the density of the independence copula. Alternatively, the entropy copula can be expressed it in terms of the $\mathrm{p}$ variate mutual information $\mathrm{T}(\mathrm{X})$ of the variables $\mathrm{X}$.

$$
H\left[c\left(u_{1}, \ldots, u_{p}\right)\right]=\sum_{i=1}^{p} H_{i}\left(x_{i}\right)-H(X)=-T(X)
$$

Therefore, the absolute value of entropy copula can be understood as a metric of statistical dependence for the variables $\mathrm{X}$. Given that $0 \leq T(X)<\infty$, it implies that the entropy copula is $-\infty<H\left[c\left(u_{1}, \ldots, u_{p}\right)\right] \leq 0$. Namely, entropy copula is always negative.

Alternatively, it can be expressed as:

$$
H(X)=\sum_{i=1}^{p} H_{i}\left(x_{i}\right)+H\left[c\left(u_{1}, \ldots, u_{p}\right)\right]
$$

In this form, the entropy copula is interpreted as measure of reduction of independence measured by entropy of independent variables $\sum_{i=1}^{p} H_{i}\left(x_{i}\right)$

\section{The Entropy Copula of the Multivariate $t$ distribution}

\section{The Multivariate t-distribution:}

According that $\mu=0$ or $\mu \neq 0$, the distribution is central or non-central respectively (Kotz \& Nadarajah, 2004). The probability density function of $p$-variate $t$ random variable with $n$ degrees of freedom and matrix $\mathrm{R}$ is given by: 
$f(t ; \mu, R)=\frac{\Gamma\left[\frac{v+p}{2}\right]}{\Gamma\left[\frac{v}{2}\right]}|v \pi R|^{-1 / 2}\left[1+\frac{(t-\mu)^{\prime} R^{-1}(t-\mu)}{v}\right]^{-\frac{v+p}{2}}$

In general, any moment $\mathrm{k}$ exists whenever $v>\mathrm{k}$. For the particular case of $v=1$, the multivariate $\mathrm{t}$ distribution becomes the multivariate Cauchy distribution

\section{Entropy Copula for the multivariate central case:}

The expression for $\mathrm{T}(\mathrm{X})$ for the multivariate $\mathrm{t}$ distribution with $\mu=0$ was given in GuerreroCusumano (1996), using $H\left[c\left(u_{1}, \ldots, u_{p}\right)\right]=-T(X)$, namely, substituting in the entropy copula density $H\left[c\left(u_{1}, \ldots, u_{p}\right) ;(v, R, \mu=0)\right]=\frac{1}{2} \ln |R|-\Omega$

$\Omega(v, p)=\ln \left\{\frac{\Gamma\left(\frac{p}{2}\right)\left[\beta\left(\frac{v+1}{2}, \frac{1}{2}\right)\right]^{p}}{\pi^{\frac{p}{2}} \beta\left(\frac{v+p}{2}, \frac{p}{2}\right)}\right\}+\frac{p(v+1)}{2}\left[\psi\left(\frac{v+1}{2}\right)-\psi\left(\frac{v}{2}\right)\right]-\frac{(v+p)}{2}\left[\psi\left(\frac{v+p}{2}\right)-\psi\left(\frac{v}{2}\right)\right]$

$\Omega(v, p)$ is tabulated in Guerrero-Cusumano (1996) for different combinations of $(v, \mathrm{p})$ and $0<|R| \leq 1$

In the case of multivariate t-variables are uncorrelated i.e. $R=I$, the entropy copula is given by $H\left[c\left(u_{1}, \ldots, u_{p}\right) ;(v, I, \mu=0)\right]=-\Omega . \quad \Omega$ represents a dependence measure for uncorrelated multivariate $\mathrm{t}$ distribution. For the practitioner, the entropy copula is simply the entropy of copula density $c_{t}(u ; R, v, \mu=0)$ Bouyé et al. (2000) gave the expression for $c_{t}(u ; R, v, \mu=0)$ for the standardized multivariate Student's distribution.

$$
c_{t}(u ; R, v, \mu=0)=\frac{\Gamma\left(\frac{v+p}{2}\right)\left[\Gamma\left(\frac{v}{2}\right)\right]^{p-1}\left[1+\frac{x^{\prime} R^{-1} x}{v}\right]^{-\frac{v+p}{2}}}{|R|^{\frac{1}{2}}\left[\Gamma\left(\frac{v+1}{2}\right)\right]^{p} \prod_{j=1}^{p}\left(1+\frac{x_{j}^{2}}{v}\right)^{-\frac{(v+1)}{2}}}
$$

Where the vector $\mathrm{x}$ is given by $x=\left[t_{v}^{-1}\left(u_{1}\right), \ldots, t_{v}^{-1}\left(u_{p}\right)\right]$. Figure 1 shows the bivariate Cauchy copula density with parameters $c_{t}(u ; R, v, \mu=0)$ with correlation matrix $R=\left[\begin{array}{cc}1 & .66 \\ .66 & 1\end{array}\right]$. 


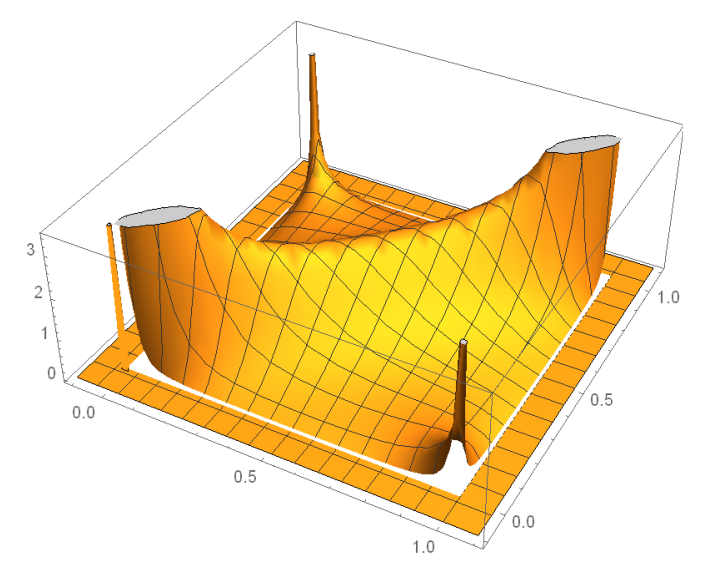

Figure 1. Bivariate Cauchy Copula Density

Also, the probability density function for the bivariate Cauchy copula distribution from the previous example (with the parameter R \& $v$ ) is given by (calculated with Mathematica):

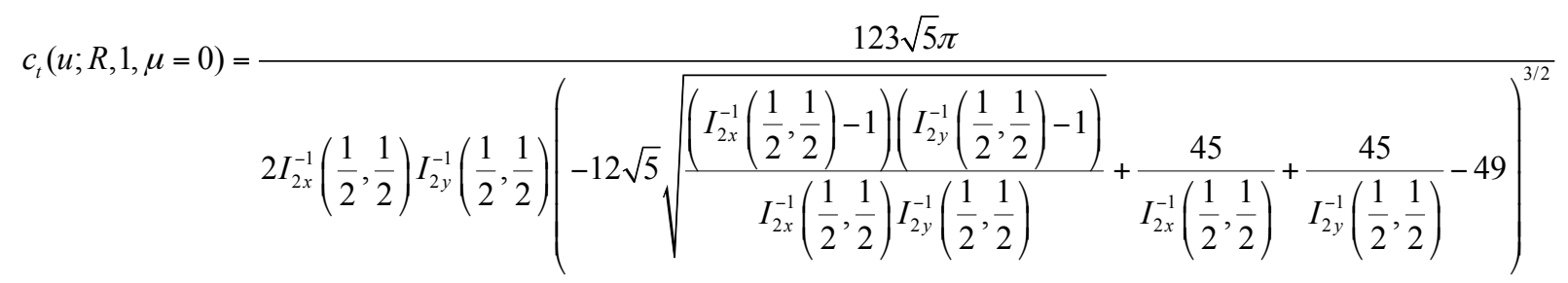

Therefore, the entropy of copula density $c(u ; R, v)$ is given by:

$$
H\left[c_{t}\left(u_{1}, \ldots, u_{p}\right) ;(v, p ; R, \mu=0)\right]=\frac{1}{2} \ln |R|-\Omega
$$

Where $H\left[c_{t}\left(u_{1}, \ldots, u_{p}\right) ;(v, p ; R, \mu=0)\right]$ represents a measure of the variability or spread of copula density $c(u ; R, v)$.

As an example, the copula density for the multivariate standardized normal (Bouyé et al., 2000) is given by $c\left(u_{1}, \ldots, u_{p}\right)=\frac{e^{-\frac{1}{2} x^{\prime} R^{-1} x}}{\mid R^{\frac{1}{2}} e^{-\frac{1}{2} \sum_{j=1}^{p} x_{j}^{2}}}$ then it is obvious that $H\left[c\left(u_{1}, \ldots, u_{p}\right) /\right.$ normal $]=0$ when the variables are uncorrelated $\mathrm{R}=\mathrm{I}$.

Also, $\Omega$ can also be understood as entropy copula excess with respect to the normal distribution, namely $H\left[C\left(u_{1}, \ldots, u_{p}\right) ;\right.$ normal $]-H\left[C\left(u_{1}, \ldots, u_{p}\right) ;(v, p ; R, \mu=0)\right]=\Omega$, which is a function degrees of freedom $v$ and $\mathrm{p}$. As the degrees of freedom $v$ decreases, then $\Omega$ increases, then the entropy copula excess is a measure of difference of fat-tailness between the multivariate $t$ and normal distribution. 


\section{The Induced Copula Variability}

A simple parametrization of the entropy copula via a transformation used for $T(X)$ is given in Guerrero-Cusumano (1996) (see also Joe, 1997), namely:

$$
0 \leq \sigma_{C_{I}}^{2}(\nu, R, \mu=0)=1-e^{2 H\left[C_{t}\left(u_{1}, \ldots, u_{p}\right) ;(v, p ; R, \mu=0)\right]} \leq 1
$$

Where $\sigma_{I}^{2}$ is defined as the induced copula variability by the copula density $c\left(u_{1}, \ldots, u_{p}\right)$. $\sigma^{2}{ }_{I}(v, p ; R, \mu=0)$ Is a measure of variability of the density copula, where a value $\sigma^{2}{ }_{I}(v, p ; R, \mu=0)$ close to 0 indicates that $\mathrm{p}$-variables are close to independence and a value close to 1 indicates total dependence. The induced copula can written variability for the multivariate $\mathrm{t}$ as: $\sigma_{C_{I}}^{2}(v, R, \mu=0)=1-|R| e^{-2 \Omega}$

In the multivariate normal, $\Omega=0$ then $\sigma_{C_{I}}^{2}$ (normal, $\left.p ; R\right)=1-|R|$. Note that when the variable are uncorrelated $R=I$ then $\sigma_{C_{I}}^{2}($ normal, $p ; R)=0$

As an example for equal correlations using the Pearson correlation coefficients:

$\sigma_{C_{I}}^{2}(v, R, \mu=0)=1-(1-\rho)^{p-1}[1+(p-1) \rho] e^{-2 \Omega}$

And for the Tau correlation coefficient

$$
\sigma_{C_{I}}^{2}(v, R, \mu=0)=1-\left[1-\sin \left(\tau \frac{\pi}{2}\right)\right]^{p-1}\left[1+(p-1) \sin \left(\tau \frac{\pi}{2}\right)\right] e^{-2 \Omega}
$$

Similarly for the Spearman correlation coefficient.

\section{Moments of the sample Induced Copula Variability_$\sigma_{C_{I}}{ }^{2}$ :}

This section provides moments and asymptotic results for the sample induced copula variability.

A possible estimator for induced copula variability is given by:

$$
\hat{\sigma}_{I}^{2}(\nu, p ; R, \mu=0)=1-e^{-2 \Omega}|\hat{R}|
$$

Where $R$ an estimator for $\mathrm{R}$ based on a sample of $\mathrm{n}$-observations from a multivariate $\mathrm{t}$ distribution. Then, the $\mathrm{j}$-moment of induced copula variability, for fixed degrees of freedom, is given by:

$$
E\left[\left(\hat{\sigma}_{I}^{2}(v, p ; R, \mu=0)\right)^{j}\right]=\sum_{k=0}^{j}(-1)^{k} e^{-2 \Omega k}\left(\begin{array}{l}
j \\
k
\end{array}\right)\left[\frac{v-2}{v(n-1)}\right]^{k} \frac{\Gamma\left(\frac{v}{2}-k p\right)}{v^{-k p} \Gamma\left(\frac{v}{2}\right)} \frac{\Gamma_{p}\left(\frac{n-1}{2}+k\right)}{\Gamma_{p}\left(\frac{n-1}{2}\right)}|R|^{k}
$$


Proof:

Based on the fact that (Gupta \& Varga, 1995; Siotani, Fujikoshi, \& Hayakawa, 1985)

$$
E\left(|\hat{R}|^{k}\right)=\left(\frac{v-2}{v(n-1)}\right)^{k} \frac{\Gamma\left(\frac{v}{2}-k p\right)}{v^{-k p} \Gamma\left(\frac{v}{2}\right)} \frac{\Gamma_{p}\left(\frac{n-1}{2}+k\right)}{\Gamma_{p}\left(\frac{n-1}{2}\right)}|R|^{k}
$$

And expressing the induced copula variability as

$$
E\left[\left(\hat{\sigma}_{I}^{2}(v, p ; R)\right)^{j}\right]=E\left[\left(1-|R| e^{-2 \Omega}\right)^{j}\right]=\sum_{k=0}^{j}(-1)^{k}\left(\begin{array}{l}
j \\
k
\end{array}\right) e^{-2 \Omega k} E\left[|R|^{k}\right]
$$

As an example, the mean and variance using the moments of $\hat{\sigma}_{I}^{2}(v, p ; R)$ is given by:

$$
E\left[\hat{\sigma}_{I}(v, p ; R)\right]=\frac{\mathrm{e}^{-4 \Omega} P^{2}(-2+v)^{2} v^{-2+2 p}\left(-\Gamma[-p+0.5 v]^{2}\left(\prod_{q=1}^{p} \Gamma\left[\frac{1}{2}(2+n-q)\right]\right)^{2}+\Gamma[-2 p+0.5 v] \Gamma[0.5 v]\left(\prod_{q=1}^{p} \Gamma\left[\frac{n-q}{2}\right]\right) \prod_{q=1}^{p} \Gamma\left[\frac{1}{2}(4+n-q)\right]\right)}{(-1+n)^{2} \Gamma[0.5 v]^{2}\left(\prod_{q=1}^{p} \Gamma\left[\frac{n-q}{2}\right]\right)^{2}}
$$

And the Variance

$$
\operatorname{Var}\left[\hat{\sigma}_{I}(v, p ; R)\right]=\frac{\mathrm{e}^{-4 \Omega} P^{2}(-2+v)^{2} v^{2}\left(-\Gamma\left[\frac{n}{2}\right]^{2} \Gamma\left[\frac{1+n}{2}\right]^{2} \Gamma[-2+0.5 v]^{2}+\Gamma\left[-1+\frac{n}{2}\right] \Gamma\left[1+\frac{n}{2}\right] \Gamma\left[\frac{1}{2}(-1+n)\right] \Gamma\left[\frac{3+n}{2}\right] \Gamma[-4+0.5 v] \Gamma[0.5 v]\right)}{(-1+n)^{2} \Gamma\left[-1+\frac{n}{2}\right]^{2} \Gamma\left[\frac{1}{2}(-1+n)\right]^{2} \Gamma[0.5 v]^{2}}
$$

Asymptotic Distribution for $\hat{H}\left[C\left(u_{1}, \ldots, u_{p}\right) ;(v, p ; R, \mu=0)\right]$

In Guerrero-Cusumano (1998) showed the asymptotic distribution of estimator of mutual information $T(X)$ for the central multivariate $t$ under different conditions assuming an unknown correlation matrix $\mathrm{R}$ and known degrees of freedom $v$. The following are results pertaining to asymptotic distribution of the entropy copula for the multivariate $t$.

Result: independent variables

The asymptotic distribution of the entropy copula for a multivariate $t$ distribution under independence is given by:

$$
(n-1) \hat{H}\left[c\left(u_{1}, \ldots, u_{p}\right) ;(v, p ; \hat{R})\right]=(n-1)\left[\frac{1}{2} \ln |\hat{R}|-\Omega\right] \stackrel{n \rightarrow \infty}{\longrightarrow} \chi_{\frac{p(p-1)}{2}}^{2}
$$

Result: Dependent variables $(R \neq I)$

The asymptotic distribution of the entropy copula for a multivariate $t$ distribution under dependence is given by: 


$$
\sqrt{n-1}\left[\hat{H}\left[c\left(u_{1}, \ldots, u_{p}\right) ;(v, p ; \hat{R})\right]-H\left[C\left(u_{1}, \ldots, u_{p}\right) ;(v, p ; R)\right] \stackrel{d}{\rightarrow} N_{\rightarrow}^{d}\left[0, \frac{\operatorname{Tr}\left(R^{2}\right)-p}{2}\right]\right.
$$

The distribution of the induced standard deviation of entropy copula is given by

$$
\sqrt{n-1}\left[\hat{\sigma}_{I}(v, p ; R)-\sigma_{I}(v, p ; R)\right]: N\left\{0, \frac{|R|^{2}\left[\operatorname{Tr}\left(R^{2}\right)-p\right] e^{-4 \Omega}}{2\left(1-e^{-2 \Omega}|R|\right)}\right\}
$$

Proof:

Olkin and Siotani (1977) showed that the asymptotic distribution of

$$
\sqrt{n-1}(|\hat{R}|-|P|): N\left\{0,2|R|^{2}\left[\operatorname{Tr}\left(R^{2}\right)-p\right]\right\}
$$

Moreover, using the delta method via Bishop's theorem (Bishop, Fienberg, \& Holland, 1975), desired result is obtained.

\section{Are the p-variate Uncorrelated?}

In practice, the practitioner may be interested controlling or determining whether the variables under study are uncorrelated but dependent, this fact is particular important in multivariate quality control (Woodall \& Montgomery, 1999; Stoumbos \& Sullivan, 2002; Vidal-Puiga \& Ferrer, 2014). The following paragraph provides an easy to implement approach to determine uncorrelatedness among $\mathrm{p}$-variate $\mathrm{t}$ distribution.

\section{Quantiles for the Entropy Copula for Uncorrelated multivariate t distribution}

A simulation study was performed to calculate the quantiles for $\hat{H}\left[C\left(u_{1}, \ldots, u_{p}\right) ;(v, p ; \hat{R})\right]$ under for an uncorrelated multivariate t, i.e. $\mathrm{R}=\mathrm{I}$ using a 1,000,000 data points with Mathematica 10 for the following parameter choices common in practice, variables $p=1(1) 5$, sample sizes $n=6(1) 20$ and degrees of freedom $v=1(1) 10$. The tables in the Appendix show the $1 \%$ and $5 \%$ quantiles respectively for different degrees of freedom and sample sizes, then any value below the chosen quantile and indicates independence for multivariate $\mathrm{p}$ model under chosen significance level.

\section{Deming's Four Principle: The Knowledge of Variation}

The knowledge of variation makes a distinction between the variation inherent and predictable to any part of the process components and the variation that it is not predictable in any part of the process components (Perla, Provost, \& Parry, 2013). Process control is the understanding of chance and assignable causes affecting the variability in a process. If a process is out of control, steps are taken to diagnose the assignable causes and remove them, thus, its purpose is fault prevention. Statistical process control and control charts are the most effective ways to address Deming's fourth principle to determine stability and control in a system. Process control seeks to 
determine whether a process is in control, meaning whether the distribution of some critical measured variable is stable. In process control, it is common to monitor and control variables that are either discrete (such as the number of defects in an item) or continuous (e.g. measurements of a diameter of a cylinder), whose distribution is assumed to be known most of the time. Traditional process control deals with one process characteristic at a time, and utilizes single-variable charts such as the X-chart and the R-chart. Control charts are control diagrams to determine whether the process is in statistical control. The construction of control limits can be computed in the following ways:

- $\quad$ Specified multiple $(\mathrm{k})$ of the standard error of $\mathrm{X}$ above and below the central line, e.g. if $\mathrm{k}=3$, these are referred to as $3 \sigma$ limits.

- Probability limits defined in terms of $\alpha$, a specified probability that X exceeds the limits.

The general multivariate statistical quality control problem considers a repetitive process where each data-item is characterized by a vector $\mathrm{X}$ of $\mathrm{p}$-interrelated variables. Because of the chance causes inherent in the process, these quality characteristics are random variables. One of the most powerful tools in the arsenal of quality control practitioners is the statistical quality control chart. In the past, all the theoretical efforts were on the creation and study of univariate control charts, but later the analysis of more complex systems created the need for multivariate control charts (Woodall \& Montgomery, 1999). The basic assumption for multivariate control charts is multivariate normality, which is completely specified by its expected value $\mu$ and covariance matrix $\Sigma$. In general, this assumption many times cannot be supported by real data (Nidsunkid Borkowski, \& Budsaba, 2017). This study will consider the underlying distribution of the observation is the multivariate $\mathrm{t}$ distribution. The $\mathrm{t}$ distribution plays an important role not only in statistics but also in different areas of business and engineering. Dunnett and Sobel (1954) encountered this distribution in the context of certain multiple decision problems. Whereas, Wang and Ip (2003) generalized the multiple linear regression models for the case that the errors follow a multivariate $t$ distribution.

\section{Example}

Opel $\mathrm{GmbH}$ is a German car manufacturer of long tradition, being founded in 1863 by Adam Opel in Rüsselsheim, Hesse, Germany. General Motors bought Opel in 1929. Opel is now part of the French conglomerate PSA. The Opel Vectra was a family car, which was introduced in October 1988 as a replacement to the Opel Ascona, and the Opel Insignia replaced it in November 2008. Car safety was always important for Opel. The company created the famous slogan "Don't start without a seatbelt", which was launched in Germany in 1974 to persuade drivers to wear seatbelts, a revolutionary idea at that time. Two years later, wearing seatbelts became compulsory and decade later, in 1984, fines for not wearing seatbelts were introduced (Press Room Europe, 2014).

Car safety is more complex than wearing a seatbelt. According to the European Parliament (2006), car safety involves three categories of body parts in the automotive sector, namely:

Category No. 1: Body panels, such as front and rear bumpers

Category No. 2: Front and rear lamps, as well as integrated mirrors 
Category No. 3: Automotive glass, in particular windscreens

Bumpers in a car are supposed to limit damage in minor collisions and protect pedestrians from injury. Bumper contacts lead to $64 \%$ of adult leg injuries. In order for the vehicle to provide adequate occupant protection in frontal crashes, portions of the vehicle bumper structure have to be stiff enough to enable the vehicle to absorb a sufficient amount of the impact energy (United Nations, 2009). A misplaced bumper fails to absorb energy and redistribute it less efficiently in a case of a crash, thereby increasing the amount of damage done to components deeper into the energy-absorbing and protective structures, also in case the car suffers a second crash, it causes worse and more expensive damage (European Parliament, 2006). Figure 2 refers to the body coordinates links for an Opel Vectra 1997 bumper, as shown in.
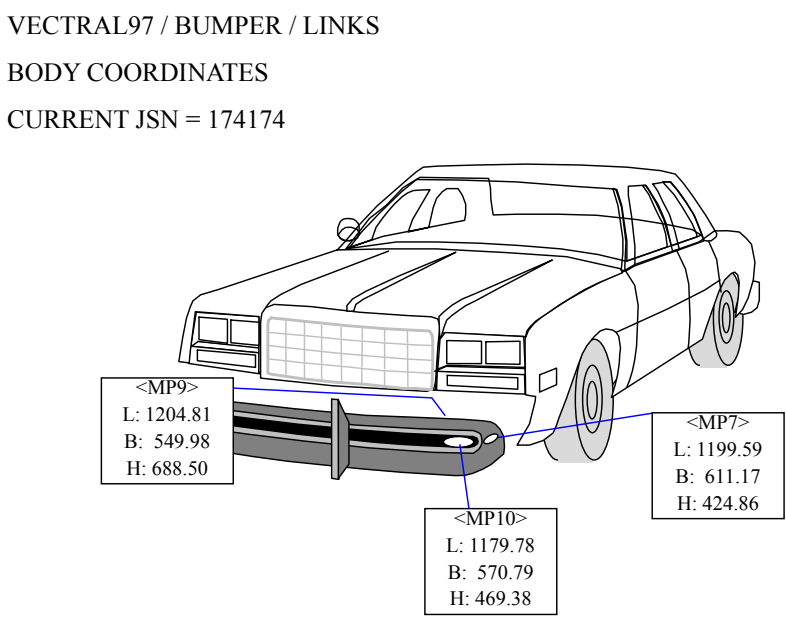

Figure 2. The body coordinates links for an Opel Vectra 1997 bumper

In each side, right and left, there are three links, denoted by MP7, MP9, and MP10. Each link is determined by three positioning dimensions, namely $\mathrm{L}, \mathrm{B}$, and $\mathrm{H}$. As an example, MPR9, i.e. MP9 in the right side: the data analyzed are the deviation for the factory target for MPR9, namely (L: 1204.81, B: 549.98 , \& H: 688.50) as shown in Figure 2. The adequacy of the multivariate $t$ approach is attested in Table 1 which shows the p-values for MP9R testing for the multivariate normal using Henze-Zirkler Test (SYSTAT software) and multivariate t distribution using the Kolmogorov-Smirnov test (Mathematica software).

Table 1. Tests for Multivariate Normality and t-ness

\begin{tabular}{|l|c|c|c|c|}
\hline Dimensions & $\begin{array}{c}\text { Multivariate Normality } \\
\text { P-value (systat) }\end{array}$ & Normality? & $\begin{array}{c}\text { Multivariate t-ness } \\
\text { p-value }\end{array}$ & T-ness? \\
\hline MP9R & 0.004 & NO & 0.815 & YES \\
\hline
\end{tabular}


Based on the results of Table 1 ( $\mathrm{p}$-value for t-ness $=0.815)$, the suggested model is a tri-variate Student $t$ distribution given by:

$$
f(t ; \mu, R)=\frac{\Gamma\left[\frac{v+p}{2}\right]}{\Gamma\left[\frac{v}{2}\right]}|v \pi R|^{-1 / 2}\left[1+\frac{(t-\mu)^{\prime} R^{-1}(t-\mu)}{v}\right]^{-\frac{v+p}{2}}
$$

The correlation matrix $\mathrm{R}$ is defined by:

$$
R=\left(\begin{array}{ccc}
1 & \rho_{12} & \rho_{13} \\
\rho_{21} & 1 & \rho_{23} \\
\rho_{31} & \rho_{32} & 1
\end{array}\right)
$$

As a quality control requirement the deviations from positioning dimensions should be uncorrelated, i.e. the matrix $\mathrm{R}$ should be the identity matrix. To simplify the parameter space, namely $\left(\mu_{1}, \mu_{2}, \mu_{3}, \rho_{1}, \rho_{2}, \rho_{3}, v\right)$, the data obtaining the following parameters should be standardized to be estimate $\left(\rho_{1}, \rho_{2}, \rho_{3}, v\right)$. In order to determine uncorrelatedness, a sample of 200 bumpers is taken. The parameters' estimation was performed using the log-likelihood function of the trivariate $t$ and the Quasi-Newton method provided by a subroutine created in SYSTAT. This

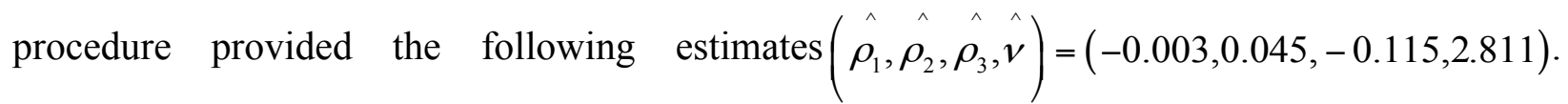
Equivalently, in matrix form for estimate correlation matrix as:

$$
\hat{R}=\left(\begin{array}{ccc}
1 & r_{12} & r_{13} \\
r_{21} & 1 & r_{23} \\
r_{31} & r_{32} & 1
\end{array}\right)=\left(\begin{array}{ccc}
1 & -.003 & .045 \\
-.003 & 1 & -.115 \\
.045 & -.115 & 1
\end{array}\right)
$$

Recall that the entropy of copula density $c(u ; R, v)$ is given by:

$$
H\left[c_{t}\left(u_{1}, \ldots, u_{p}\right)\right]=\frac{1}{2} \ln |R|-\Omega
$$

In this case, $-\hat{H}\left[c\left(u_{1}, \ldots, u_{p}\right)\right]=\left[\Omega(\hat{v}, p)-\frac{1}{2} \ln |\hat{R}|\right]=(0.587255-.5 * 0.984772)=0.0948686$ which is interpreted as a measure of reduction of independence in a system defined by 3-related positioning $\mathrm{L}, \mathrm{B}$, and $\mathrm{H}$.

\section{Are the Variables Uncorrelated?}

A factory quality control requirement is to determine the uncorrelatedness of vector $\mathrm{X}$, which is equivalent to $H\left[c_{t}\left(u_{1}, \ldots, u_{p}\right) ; R=I\right]=-\Omega$. Assume that in Phase 1 of the statistical process control, the sample $n=200$, corresponds to 10 samples of size $n=20$ observed through time. For 
each sample the value of $v$ and $\hat{H}\left[c\left(u_{1}, \ldots, u_{p}\right)\right](\mathrm{i}=1,2 \ldots 10)$ was calculated. Table 2 provides with the value of $\hat{v}$ and $\hat{H}\left[c\left(u_{1}, \ldots, u_{p}\right)\right]$.

Table 2. Entropy Copula

\begin{tabular}{|l|c|c|c|c|c|c|c|c|c|c|}
\hline Sample & $\mathbf{1}$ & $\mathbf{2}$ & $\mathbf{3}$ & $\mathbf{4}$ & $\mathbf{5}$ & $\mathbf{6}$ & $\mathbf{7}$ & $\mathbf{8}$ & $\mathbf{9}$ & $\mathbf{1 0}$ \\
\hline$\hat{\boldsymbol{v}}$ & 2.904 & 2.366 & 2.928 & 2.609 & 3.215 & 2.932 & 2.664 & 2.496 & 3.031 & 2.554 \\
$\hat{\wedge} \hat{-H}$ & 0.144456 & 0.227462 & 0.114409 & 0.134616 & 0.103121 & 0.182037 & 0.162844 & 0.156987 & 0.075347 & 0.233828 \\
\hline
\end{tabular}

In practice, the average value of $v$ as $v \approx 3$ (p-value 0.032 ) is considered. As an example for sample 1 in the table, the parameters for this sample were calculated as $\left(\hat{\rho_{1}}, \hat{\rho_{2}}, \hat{\rho_{3}} ; \hat{v}\right)=(-0.043,-0.056,-0.36 ; 2.904)$, then $-\hat{H}\left[c\left(u_{1}, \ldots, u_{p}\right)\right]=0.144456$, namely 0.144456 is the entropy copula for that sample. To determine whether the variables are correlated or not for each sample, consider an $\alpha=5 \%$, table gives the $95 \%$ quantiles for the distribution of $\hat{H}\left[c\left(u_{1}, \ldots, u_{p}\right)\right]$, for the situation when $\mathrm{p}=3, \mathrm{n}=20$, and $v=3$, the $95 \%$ quantile is given by $H_{95 \%}(n=3, v=3, n=20)=-0.3562$ and similarly for $H_{95 \%}(n=3, v=2, n=20)=-0.4560$. Comparing the cut-off points with value of $\hat{H}\left[c\left(u_{1}, \ldots, u_{p}\right)\right]$ given in the table, all samples shows variables that are uncorrelated. This shows that the system is in statistical process control for the correlation structure for a multivariate $t$ model. Such fact is very relevant in process control when it is expected or required that the different deviations should be uncorrelated to avoid any special causes in the installation of car bumpers.

\section{Independence}

Control requirements in this example was for uncorrelatedness of (L, B, \& H), which only measures the linear dependence among $(\mathrm{L}, \mathrm{B}, \& \mathrm{H})$. The quality control expert could be interested in a strong requirement such as independence. In order to address to the question whether the three positioning dimensions, namely $(\mathrm{L}, \mathrm{B}, \& \mathrm{H})$, are independent? The asymptotic distribution of the entropy copula for the whole 200 bumpers, namely:

$$
-(n-1) \hat{H}\left[c\left(u_{1}, \ldots, u_{p}\right) ;(v, p ; \hat{R})\right]=(n-1)\left[\Omega-\frac{1}{2} \ln |\hat{R}|\right] \stackrel{n \rightarrow \infty}{\longrightarrow} \chi_{\frac{p(p-1)}{2}}^{2}
$$

Therefore, 


$$
\begin{aligned}
& -(n-1) \hat{H}\left[c\left(u_{1}, \ldots, u_{p}\right)\right]=(200-1)\left[\Omega(\hat{v}, p)-\frac{1}{2} \ln |\hat{R}|\right]= \\
& 199 *(0.587255-.5 * 0.984772)=199 * 0.0948686=18.87
\end{aligned}
$$

The asymptotic control limits to determine independence are determine by:

$$
U C L=\chi_{\left(\frac{p(p-1)}{2} ; 1-\alpha\right)}^{2}=\chi_{\left(\frac{3(3-1)}{2} ; 0.95\right)}^{2}=7.48442
$$

Therefore, at $\alpha=5 \%$, the three positioning dimension deviations are dependent. The quality control expert should reconsider whether uncorrelatedness should be sufficient for quality control. Given that the measurements are dependent it will created future quality control problems if they are not addressed.

\section{Concluding Remarks}

Deming's SPK is a scientific framework where one of its basic principles is the study of variation. Complex systems may vary through time and their variability can be measured by its entropy as well as its interraledness by its entropy copula. The entropy copula could be interpreted as a measure of reduction of independence in a system defined by p-related variables. This paper introduces the concept of entropy copula and induced entropy copula for the multivariate central to measure as well as quantify the degrees of dependence via the entropy copula formula. These copulas measures are represented via various correlation coefficients, which may be useful for practitioners. This research derived the moments of the induced entropy copula and also develop asymptotics for distributions for the entropy copula under various scenarios as well as provide tables to test uncorrelatedness for multivariate central $t$ distribution for $\alpha=5 \%$ and $\alpha=1 \%$.

In practice, there are situations in which the simultaneous control of two or more related/ unrelated quality characteristics is mandatory. Process-monitoring problems in which several related/unrelated variables are called multivariate quality-control problems (Woodall \& Montgomery, 1999). The theory and techniques developed in this paper can be valuable to create multivariate control mechanism to measure dependence or lack of dependence in a multivariate control system for the car or other related industries. The example also shows how entropy copula could be used as a measure of reduction of independence in a system defined by p-related variables.

\section{References}

Bishop, Y., Fienberg S., \& Holland, P. (1975). Discrete multivariate analysis: Theory and practice. Cambridge, MA: MIT Press.

Bouyé, E., Durrleman, V., Nikeghbali, A., Riboulet, G., \& Roncalli, T. (2000). Copulas for finance-a reading guide and some applications. Groupe de Recherche Operationnelle, Credit Lyonnais Paris. 
Burnes, B. (2004). Kurt Lewin and complexity theories: Back to the future? Journal of Change Management, 4(4), 309-325.

Czado, C. (2010). Pair-copula constructions of multivariate copulas. In Copula theory and its applications (pp. 93-109). Berlin, Heidelberg: Springer.

Delahaye, B. L. (2002) The management of knowledge: A systems theory approach for vocational education and training. In Searle, Jean \& Roebuck, Dick (Eds.) 10th Annual International Conference on Post-Compulsory Education and Training, Surfers Paradise.

Deming, W. E. (1986). Out of the Crisis. Cambridge, MA: MIT Press - Center for Advanced Educational Services.

Deming, W. E. (1994). The new economics: For industry, government, education. Cambridge, MA: MIT Press - Center for Advanced Educational Services.

Díaz-Viera, M., \& Casar-González, R. (2005). Stochastic simulation of complex dependency patterns of petrophysical properties using t-copulas (Vol. 2). In Proceedings of the International Association for Mathematical Geosciences GIS and Spatial Analysis (pp. 749-755).

Dunnett, C. W., \& Sobel, M. (1954). A bivariate generalization of Student's t-distribution, with tables for certain special cases. Biometrika, 41(1-2), 153-169.

Edgeman, R. L., \& Fraley, L. A. (2008). A system of profound consciousness: Building beyond Deming. Total Quality Management, 19(7-8), 683-707.

Embrechts, P. (2009). Copulas: A personal view. Journal of Risk and Insurance, 76(3), 639-650.

European Parliament (2006). The consequences for the safety of consumers and third parties of the proposed directive amending directive 98/7/1 on legal protection of design rights (2006). Brussels. Retrieved from: http://www.europarl.europa.eu/RegData/ etudes/etudes/join/2006/378261/IPOL-JOIN_ET(2006)378261_EN.pdf

Fernández, M., \& Kolev, N. (2007). Bivariate density classification by the geometry of the marginals. Economic Quality Control, 22(1), 3-18.

Guerrero, J. L. (1994). Multivariate mutual information: Sampling distribution with applications. Communications in Statistics-Theory and Methods, 23(5), 1319-1339.

Guerrero-Cusumano, J. L. (1996). A measure of total variability for the multivariate $\mathrm{t}$ distribution with applications to finance. Information Sciences, 92(1-4), 47-63.

Guerrero-Cusumano, J. L. (1998). Measures of dependence for the multivariate t distribution with applications to the stock market. Communications in Statistics-Theory and Methods, 27(12), 2985-3006.

Gupta, A. K., \& Varga, T. (1993). Linear models. In Elliptically contoured models in statistics (pp. 285-310). Dordrecht, Netherlands: Springer. 
Hensler, D. A., Edgeman, R. L., \& Guerrero-Cusumano, J. L. (2000). East meets west: weaving the threads of Deming, da Vinci and the Tao Te Ching. Total Quality Management, 11(46), 501-508.

Höffding, W. (1940). Masstabinvariante korrelationstheorie. Schriften des Mathematischen Instituts und Instituts fur Angewandte Mathematik der Universitat Berlin, 5, 181-233.

Jaworski, P., Durante, F., Härdle, W. K., \& Rychlik, T. (Eds.) (2010). Copula theory and its applications. In proceedings of the workshop held in Warsaw (Vol. 198). Berlin, Germany: Springer Science \& Business Media.

Joe, H. (1997). Multivariate models and multivariate dependence concepts. Boca Raton, FL: CRC Press.

Jöhri, S. (2004). Portfolio optimization with hedge funds: Conditional value at risk and conditional draw-down at risk for portfolio optimization with alternative investments (Doctoral dissertation, ETH, Eidgenössische Technische Hochschule Zürich, Department of Computer Science).

Kotz, S., \& Nadarajah, S. (2004). Multivariate t-distributions and their applications. Cambridge, United Kingdom: Cambridge University Press.

Kullback, S. (1968). Information theory and statistics. Chelmsford, MA: Courier Corporation.

Lambert, P. (2007). Archimedean copula estimation using Bayesian splines smoothing techniques. Computational Statistics \& Data Analysis, 51(12), 6307-6320.

Nelsen, R. (2006). An introduction to copulas. ( $2^{\text {nd }}$ Ed.). Berlin, Germany: Springer.

Nidsunkid, S., Borkowski, J. J., \& Budsaba, K. (2017). The effects of violations of the multivariate normality assumption in multivariate Shewhart and MEWMA control charts. Quality and Reliability Engineering International, 33(8), 2563-2576.

Olkin, I., \& Siotani, T. (1977). Asymptotic distribution of functions of a correlation matrix. In Essays in Probability and Statistics (ed. S. Ikeda, et al.), Shinko-Tsusho, Tokyo, pp. 235251.

Perla, R. J., Provost, L. P., \& Parry, G. J. (2013). Seven propositions of the science of improvement: Exploring foundations. Quality Management in Healthcare, 22(3), 170186.

Press Room Europe (2014). Uwe Mertin, 40 years of opel safety vehicle: The safe route from Kadett C to Astra. Retrieved from: https://media.gm.com/media/intl/en/opel/home.detail. html/content/Pages/news/intl/en/2014/opel/09-23-safety-vehicle.html

Savu, C., \& Trede, M. (2010). Hierarchies of Archimedean copulas. Quantitative Finance, 10(3), 295-304.

Sklar, M. (1959). Fonctions de repartition an dimensions et leurs marges. Publications de l'Institut de statistique de l'Université de Paris . Paris, 8, 229-231. 
Siotani, T., Fujikoshi, Y., \& Hayakawa, T. (1985). Modern multivariate statistical analysis, a graduate course and handbook. Columbus, OH: American Sciences Press.

Stacey, R. D. (2007). Strategic management and organisational dynamics: The challenge of complexity to ways of thinking about organisations. London, United Kingdom: Pearson Education.

Stoumbos, Z. G., \& Sullivan, J. H. (2002). Robustness to non-normality of the multivariate EWMA control chart. Journal of Quality Technology, 34(3), 260-276.

Sutradhar, B. C., \& Ali, M. M. (1986). Estimation of the parameters of a regression model with a multivariate t error variable. Communications in Statistics-Theory and Methods, 15(2), 429-450.

United Nations (2009). Global technical regulation No. 9, Pedestrian Safety. Retrieved from: https://www.unece.org/fileadmin/DAM/trans/main/wp29/wp29wgs/wp29gen/wp2 9registry/ECE-TRANS-180a9e.pdf

Vidal-Puig, S., \& Ferrer, A. (2014). A comparative study of different methodologies for fault diagnosis in multivariate quality control. Communications in Statistics-Simulation and Computation, 43(5), 986-1005.

Wang, S. G., \& Ip, W. C. (2003). Inconsistency of estimate of the degree of freedom of multivariate student-t disturbances in linear regression models. Economics Letters, 80(3), 383-389.

Woodall, W. H., \& Montgomery, D. C. (1999). Research issues and ideas in statistical process control. Journal of Quality Technology, 31(4), 376-386.

\section{Author's Biography}

Dr. Guerrero-Cusumano is a Tenured Associate Professor at the Georgetown University School of Business. He holds a Ph.D. in Industrial Engineering from the University of Illinois and a Master of Sciences in Statistics degree from the Mathematics Department of the University of Illinois and he is also an Economics Statistician from the School of Economics Science, Rosario University, Argentina. Professor Guerrero-Cusumano was the former academic director of the Corporate International Master's at the Georgetown University School of Business. He was former Director of the International Institute on Government, Management, and Policy at Georgetown U. Prof. Guerrero-Cusumano has been a member of the faculty at Georgetown since 1989 in the School of Business. He is also the recipient of the Gold Medal for service at Georgetown University. In 2008, Professor Guerrero-Cusumano was awarded an Honorary Doctorate, Doctor Honoris Causa in Administration by Ovidius University (Romania). Also in 2008, he has been elected a Fellow at the Judge Business School, Cambridge University, Great Britain. He was also conferred and nominated for different teaching and research awards 


\section{Appendix}

Table 3. Quantiles for $\hat{H}\left[C\left(u_{1}, \ldots, u_{p}\right) ;(v, p ; R=I ; \Delta=0)\right]$ with uncorrelated multivariate $\mathrm{t}$ distribution for different degrees of freedom $v$, number of variables $\mathrm{p}$ and samples sizes $\mathrm{n}$ at $5 \%$.

\begin{tabular}{|c|c|c|c|c|c|c|c|c|c|c|c|}
\hline $5 \%$ & $v=1$ & $v=2$ & $v=3$ & $v=4$ & $v=5$ & $v=6$ & $v=7$ & $v=8$ & $v=9$ & $v=10$ & $\mathbf{n}$ \\
\hline \multirow[t]{15}{*}{$\mathrm{p}=2$} & -0.85483 & -0.71371 & -0.67474 & -0.65408 & -0.65299 & -0.64113 & -0.64454 & -0.63510 & -0.63866 & -0.63435 & 6 \\
\hline & -0.71720 & -0.57588 & -0.52702 & -0.51961 & -0.51007 & -0.50282 & -0.50154 & -0.50091 & -0.49700 & -0.49673 & 7 \\
\hline & -0.62763 & -0.48510 & -0.44435 & -0.43284 & -0.41854 & -0.41835 & -0.40949 & -0.41198 & -0.40869 & -0.40739 & 8 \\
\hline & -0.56502 & -0.42281 & -0.38233 & -0.36547 & -0.35911 & -0.34995 & -0.34683 & -0.34673 & -0.34793 & -0.34500 & 9 \\
\hline & -0.51746 & -0.37487 & -0.33477 & -0.32028 & -0.30975 & -0.30396 & -0.30248 & -0.29836 & -0.29598 & -0.29676 & 10 \\
\hline & -0.47801 & -0.33942 & -0.29890 & -0.28339 & -0.27498 & -0.27337 & -0.26482 & -0.26214 & -0.26382 & -0.26440 & 11 \\
\hline & -0.45201 & -0.31136 & -0.27083 & -0.25487 & -0.24556 & -0.24160 & -0.23656 & -0.23462 & -0.23511 & -0.23363 & 12 \\
\hline & -0.43025 & -0.28939 & -0.24768 & -0.22952 & -0.22412 & -0.21739 & -0.21617 & -0.21175 & -0.20954 & -0.21150 & 12 \\
\hline & -0.41176 & -0.27032 & -0.22837 & -0.21256 & -0.20307 & -0.19815 & -0.19712 & -0.19633 & -0.19425 & -0.19062 & 14 \\
\hline & -0.39628 & -0.25314 & -0.21466 & -0.19700 & -0.18838 & $\begin{array}{l}-0.18109 \\
\end{array}$ & $\begin{array}{l}-0.17991 \\
\end{array}$ & -0.17861 & -0.17720 & -0.17540 & 15 \\
\hline & -0.38193 & -0.24045 & -0.20057 & -0.18093 & -0.17426 & -0.16797 & -0.16785 & -0.16495 & -0.16309 & -0.16303 & 16 \\
\hline & -0.37124 & -0.22934 & -0.18808 & -0.17066 & -0.16430 & -0.15690 & -0.15577 & -0.15343 & -0.15229 & -0.15051 & 17 \\
\hline & -0.36168 & -0.21931 & -0.17809 & -0.16121 & -0.15330 & -0.14799 & -0.14577 & -0.14188 & -0.14117 & -0.13984 & 18 \\
\hline & -0.35069 & -0.21068 & -0.16968 & -0.15234 & -0.14508 & -0.14031 & -0.13550 & -0.13335 & -0.13261 & -0.13134 & 19 \\
\hline & -0.34337 & -0.20166 & -0.16231 & -0.14454 & -0.13695 & -0.13294 & -0.12876 & -0.12575 & -0.12494 & -0.12445 & 20 \\
\hline \multirow[t]{15}{*}{$p=3$} & -1.93428 & -1.61308 & -1.52245 & -1.47344 & -1.45232 & -1.43898 & -1.43558 & -1.43219 & -1.42044 & -1.42624 & 6 \\
\hline & -1.60810 & -1.28312 & -1.18483 & -1.13374 & -1.11418 & -1.10172 & -1.09518 & -1.09219 & -1.08743 & -1.08316 & 7 \\
\hline & -1.39145 & -1.07434 & -0.98116 & -0.93293 & -0.91081 & -0.90311 & -0.88736 & -0.88160 & -0.87842 & -0.87804 & 8 \\
\hline & -1.25622 & -0.93046 & -0.83299 & -0.79145 & -0.76963 & -0.75079 & -0.74407 & -0.74500 & -0.74198 & -0.73599 & 9 \\
\hline & -1.15629 & -0.82840 & -0.73092 & -0.68495 & -0.65944 & -0.65077 & -0.64534 & -0.63889 & -0.62981 & -0.63049 & 10 \\
\hline & -1.07248 & -0.74409 & -0.64715 & -0.60547 & -0.58927 & -0.57459 & -0.56661 & -0.55967 & -0.55332 & -0.55386 & 11 \\
\hline & -1.00573 & -0.68373 & -0.58931 & -0.54954 & -0.52755 & -0.51371 & -0.50043 & -0.50098 & -0.49802 & -0.48979 & 12 \\
\hline & -0.95708 & -0.63682 & -0.53783 & -0.49767 & -0.47678 & -0.46154 & -0.45541 & -0.45149 & -0.44732 & -0.44230 & 12 \\
\hline & -0.92034 & -0.59722 & -0.49929 & -0.45783 & -0.43279 & -0.42247 & -0.41423 & -0.41004 & -0.40997 & -0.40332 & 14 \\
\hline & -0.88437 & -0.56247 & -0.46481 & -0.42588 & -0.40013 & -0.38680 & -0.38069 & -0.37422 & -0.37184 & -0.36856 & 15 \\
\hline & -0.85788 & -0.53448 & -0.43781 & -0.39456 & -0.37238 & -0.36181 & -0.35121 & -0.34427 & -0.34092 & -0.33852 & 16 \\
\hline & -0.83311 & -0.50913 & -0.41450 & -0.36845 & -0.35062 & -0.33765 & -0.32765 & -0.32127 & -0.31930 & -0.31622 & 17 \\
\hline & -0.81285 & -0.48966 & -0.39272 & -0.35087 & -0.32771 & -0.31409 & -0.30566 & -0.30004 & -0.29853 & -0.29363 & 18 \\
\hline & -0.79313 & -0.47125 & -0.37407 & -0.33265 & -0.31088 & -0.29736 & -0.28951 & -0.28321 & -0.27912 & -0.27901 & 19 \\
\hline & -0.77778 & -0.45601 & -0.35620 & -0.31545 & -0.29523 & -0.28105 & -0.27291 & -0.26795 & -0.26347 & -0.25976 & 20 \\
\hline \multirow[t]{2}{*}{$\mathrm{p}=4$} & -3.51744 & -2.98657 & -2.83569 & -2.76446 & -2.71191 & -2.70039 & -2.68437 & -2.66813 & -2.66759 & -2.67099 & 6 \\
\hline & -2.80250 & -2.27576 & -2.10349 & -2.03728 & -1.99197 & -1.96753 & -1.95432 & -1.94653 & -1.95002 & -1.93392 & 7 \\
\hline
\end{tabular}




\section{Online Journal of Applied Knowledge Management}

A Publication of the International Institute for Applied Knowledge Management

Volume 6, Issue 1, 2018

\begin{tabular}{|c|c|c|c|c|c|c|c|c|c|c|c|}
\hline & -2.37820 & -1.85440 & -1.69288 & -1.62415 & -1.58680 & -1.56196 & -1.55393 & -1.53442 & -1.53054 & -1.52594 & 8 \\
\hline & -2.12126 & -1.59356 & -1.43462 & -1.35848 & -1.32395 & -1.30503 & -1.27679 & -1.26886 & -1.26380 & -1.26341 & 9 \\
\hline & -1.92862 & -1.40718 & -1.24614 & -1.17137 & -1.12608 & -1.11078 & -1.08776 & -1.08745 & -1.07601 & -1.07105 & 10 \\
\hline & -1.78476 & -1.26106 & -1.10552 & -1.03130 & -0.99204 & $\begin{array}{l}-0.97599 \\
\end{array}$ & -0.95757 & -0.94473 & -0.93316 & -0.93099 & 11 \\
\hline & -1.68129 & -1.15540 & -0.99485 & -0.92472 & -0.88688 & -0.86176 & -0.84591 & -0.83874 & -0.83204 & -0.82533 & 12 \\
\hline & -1.59606 & -1.07671 & -0.91315 & -0.83853 & -0.80120 & -0.77389 & -0.76234 & -0.75101 & -0.74626 & -0.74358 & 12 \\
\hline & -1.52660 & -1.00446 & -0.84285 & -0.76950 & -0.72534 & -0.71201 & -0.69286 & -0.68221 & -0.67596 & -0.67470 & 14 \\
\hline & -1.47065 & -0.94656 & -0.78173 & -0.71067 & -0.67503 & -0.64835 & -0.63450 & -0.62575 & -0.61960 & -0.61167 & 15 \\
\hline & -1.42095 & -0.89784 & -0.73542 & -0.66348 & -0.62238 & -0.60225 & -0.58861 & -0.57635 & -0.57239 & -0.56558 & 16 \\
\hline & -1.37908 & -0.86168 & -0.69560 & -0.62345 & -0.58393 & -0.55907 & -0.54483 & -0.53836 & -0.52977 & -0.52572 & 17 \\
\hline & -1.34390 & -0.82003 & -0.66143 & -0.58806 & -0.54893 & -0.52596 & -0.51033 & -0.50180 & -0.49267 & -0.49074 & 18 \\
\hline & -1.31347 & -0.79426 & -0.62847 & -0.55657 & -0.51897 & -0.49643 & -0.47938 & -0.47061 & -0.46261 & -0.45808 & 19 \\
\hline & -1.28726 & -0.76302 & -0.60361 & -0.52902 & -0.48980 & -0.46794 & -0.45329 & -0.44232 & -0.43605 & -0.43191 & 20 \\
\hline $\mathrm{p}=5$ & -6.39974 & -5.66964 & -5.45254 & -5.31741 & -5.25114 & -5.25708 & -5.23565 & -5.21176 & -5.15653 & -5.19168 & 6 \\
\hline & -4.46764 & -3.73845 & -3.51251 & -3.40098 & -3.33541 & -3.31122 & -3.28276 & -3.27072 & -3.26952 & -3.25557 & 7 \\
\hline & -3.66157 & -2.92689 & -2.70020 & -2.59862 & -2.53814 & -2.49991 & -2.48123 & -2.45862 & -2.45582 & -2.44786 & 8 \\
\hline & -3.18298 & -2.44656 & -2.21802 & -2.11164 & -2.06122 & -2.02226 & -2.00399 & -1.98356 & -1.97540 & -1.97918 & 9 \\
\hline & -2.86660 & -2.14164 & -1.90622 & -1.79686 & -1.74491 & -1.71292 & -1.68817 & -1.67043 & -1.66319 & -1.65367 & 10 \\
\hline & -2.63718 & -1.90655 & -1.68556 & -1.56856 & -1.51368 & -1.48493 & -1.46483 & -1.44556 & -1.43150 & -1.42239 & 11 \\
\hline & -2.47372 & -1.74548 & -1.50728 & -1.40011 & -1.34123 & -1.31489 & -1.28780 & -1.28030 & -1.26591 & -1.25172 & 12 \\
\hline & -2.33091 & -1.60571 & -1.36891 & -1.26185 & -1.21305 & -1.17434 & -1.15200 & -1.13928 & -1.12364 & -1.11675 & 12 \\
\hline & -2.22529 & -1.49815 & -1.26246 & -1.16195 & -1.10320 & -1.06351 & -1.04468 & -1.02726 & -1.01732 & -1.00907 & 14 \\
\hline & -2.13697 & -1.40823 & -1.17614 & -1.06997 & -1.01172 & -0.98050 & -0.95557 & -0.94028 & -0.92905 & -0.92110 & 15 \\
\hline & -2.06158 & -1.33145 & -1.09752 & -0.99315 & -0.94233 & -0.90219 & -0.88269 & -0.86765 & -0.85489 & -0.84698 & 16 \\
\hline & -1.99378 & -1.26871 & -1.03617 & -0.93536 & -0.87549 & -0.84024 & -0.81357 & -0.79960 & -0.78888 & -0.78283 & 17 \\
\hline & -1.94549 & -1.21597 & -0.98245 & -0.88070 & -0.82021 & -0.78575 & -0.76336 & -0.74859 & -0.73661 & -0.73049 & 18 \\
\hline & -1.89795 & -1.17025 & -0.93753 & -0.82831 & -0.77187 & -0.73938 & -0.71749 & -0.70256 & -0.69112 & -0.67997 & 19 \\
\hline & -1.85556 & -1.12737 & -0.89463 & -0.79317 & -0.73140 & -0.69903 & -0.67650 & -0.65697 & -0.64788 & -0.64182 & 20 \\
\hline
\end{tabular}


Table 4. Quantiles for $\hat{H}\left[C\left(u_{1}, \ldots, u_{p}\right) ;(v, p ; R=I ; \Delta=0)\right]$ with uncorrelated multivariate $\mathrm{t}$ distribution for different degrees of freedom $v$, number of variables $\mathrm{p}$ and samples sizes $\mathrm{n}$ at $1 \%$.

\begin{tabular}{|c|c|c|c|c|c|c|c|c|c|c|c|}
\hline $1 \%$ & $\mathbf{n}$ & $v=1$ & $v=2$ & $v=3$ & $v=4$ & $v=5$ & $v=6$ & $v=7$ & $v=8$ & $v=9$ & $v=10$ \\
\hline \multirow[t]{15}{*}{$\mathrm{p}=2$} & 6 & -1.12072 & -1.10004 & -1.08436 & -1.04918 & -1.06515 & -1.04278 & -1.05408 & -1.02858 & -1.04696 & -1.26778 \\
\hline & 7 & -0.91295 & -0.85578 & -0.84573 & -0.85236 & -0.84393 & -0.83296 & -0.82399 & -0.84356 & -0.81719 & -1.05200 \\
\hline & 8 & $\begin{array}{l}-0.76941 \\
\end{array}$ & -0.71705 & -0.70594 & -0.70595 & -0.69034 & -0.68981 & -0.68375 & -0.68267 & -0.68539 & -0.90032 \\
\hline & 9 & -0.66237 & -0.61882 & -0.60217 & -0.58804 & -0.59124 & -0.58709 & -0.57122 & -0.58107 & -0.58180 & -0.79755 \\
\hline & 10 & -0.58501 & -0.53960 & -0.52387 & -0.51307 & -0.51265 & -0.51162 & -0.50207 & -0.50230 & -0.50354 & -0.72272 \\
\hline & 11 & -0.52162 & -0.48084 & -0.46188 & -0.45893 & -0.45382 & -0.44544 & -0.43800 & -0.44640 & -0.43639 & -0.65431 \\
\hline & 12 & -0.46400 & -0.43719 & -0.42135 & -0.41099 & -0.40409 & -0.40566 & -0.39613 & -0.39329 & -0.39328 & -0.61256 \\
\hline & 12 & -0.43787 & -0.39291 & -0.37822 & -0.36929 & -0.37136 & -0.35727 & -0.36379 & -0.35777 & -0.35887 & -0.57273 \\
\hline & 14 & -0.39926 & -0.36265 & -0.34246 & -0.33694 & -0.33011 & -0.32509 & -0.32240 & -0.32542 & -0.32492 & -0.53871 \\
\hline & 15 & -0.38023 & -0.33705 & -0.31876 & -0.31248 & -0.30389 & -0.30270 & -0.30078 & -0.29784 & -0.29494 & -0.51945 \\
\hline & 16 & -0.35045 & -0.31556 & -0.29679 & -0.28887 & -0.28331 & -0.27714 & -0.27495 & -0.27241 & -0.27556 & -0.49590 \\
\hline & 17 & -0.33213 & -0.29451 & -0.27563 & -0.26731 & -0.26225 & -0.25979 & -0.25744 & -0.25780 & -0.25476 & -0.47571 \\
\hline & 18 & -0.31779 & -0.27270 & -0.25922 & -0.25112 & -0.24688 & -0.24346 & -0.23792 & -0.23875 & -0.24153 & -0.45261 \\
\hline & 19 & -0.30232 & -0.26347 & -0.24171 & -0.23592 & -0.23223 & -0.22979 & -0.22697 & -0.22264 & -0.22415 & -0.44538 \\
\hline & 20 & -0.28881 & -0.24803 & -0.23154 & -0.22063 & -0.21959 & -0.21545 & -0.21461 & -0.21299 & -0.21041 & -0.42863 \\
\hline \multirow[t]{15}{*}{$\mathrm{p}=3$} & 6 & -2.19370 & -2.10900 & -2.04712 & -2.06107 & -2.03767 & -2.02814 & -2.02991 & -2.01263 & -2.00928 & -2.54873 \\
\hline & 7 & -1.74167 & -1.62973 & -1.61055 & -1.57976 & -1.56388 & -1.55336 & -1.55877 & -1.54006 & -1.55706 & -2.07787 \\
\hline & 8 & -1.43676 & -1.36003 & -1.30636 & -1.29349 & -1.26017 & -1.26108 & -1.25860 & -1.25932 & -1.24017 & -1.77304 \\
\hline & 9 & -1.24486 & -1.15247 & -1.09869 & -1.07462 & -1.07593 & -1.05656 & -1.05542 & -1.05866 & -1.04733 & -1.56736 \\
\hline & 10 & -1.10051 & -0.98998 & -0.95930 & -0.93631 & -0.91556 & -0.91173 & -0.90612 & -0.90339 & -0.90946 & -1.41737 \\
\hline & 11 & -0.99133 & -0.89053 & -0.84081 & -0.82012 & -0.81057 & -0.79720 & -0.79326 & -0.79090 & -0.79098 & -1.30609 \\
\hline & 12 & -0.89330 & -0.80050 & -0.75948 & -0.73523 & -0.72388 & -0.71726 & -0.71273 & -0.70801 & -0.71674 & -1.22306 \\
\hline & 12 & -0.82481 & -0.72950 & -0.68327 & -0.66508 & -0.65448 & -0.64815 & -0.64150 & -0.63478 & -0.63142 & -1.15532 \\
\hline & 14 & -0.76993 & -0.67132 & -0.63185 & -0.61208 & -0.59778 & -0.57745 & -0.57798 & -0.57287 & -0.57400 & -1.09430 \\
\hline & 15 & -0.71956 & -0.62201 & -0.58608 & -0.55761 & -0.54928 & -0.54477 & -0.53300 & -0.53066 & -0.52600 & -1.04716 \\
\hline & 16 & -0.67602 & -0.58320 & -0.54450 & -0.51683 & -0.51018 & -0.49878 & -0.48814 & -0.48773 & -0.48607 & -1.00258 \\
\hline & 17 & -0.64691 & -0.54594 & -0.50674 & -0.48086 & -0.46825 & -0.45999 & -0.45615 & -0.45560 & -0.45178 & -0.96706 \\
\hline & 18 & -0.61734 & -0.51863 & -0.47392 & -0.45881 & -0.44426 & -0.43376 & -0.42985 & -0.42456 & -0.42012 & -0.93633 \\
\hline & 19 & -0.59152 & -0.49177 & -0.44577 & -0.42891 & -0.41517 & -0.40544 & -0.39883 & -0.39660 & -0.39540 & -0.91299 \\
\hline & 20 & -0.56710 & -0.46937 & -0.42684 & -0.40246 & -0.38845 & -0.38448 & -0.37584 & -0.37227 & -0.37080 & -0.88871 \\
\hline \multirow[t]{4}{*}{$\mathrm{p}=4$} & 6 & -3.90389 & -3.71943 & -3.64010 & -3.63273 & -3.59310 & -3.56897 & -3.56812 & -3.56173 & -3.57118 & -4.42559 \\
\hline & 7 & -2.89384 & -2.73729 & -2.67418 & -2.63814 & -2.61364 & -2.60558 & -2.58243 & -2.56560 & -2.54968 & -3.42334 \\
\hline & 8 & -2.36647 & -2.20060 & -2.10882 & -2.08159 & -2.06221 & -2.03550 & -2.03847 & -2.03675 & -2.01663 & -2.87223 \\
\hline & 9 & -2.00949 & -1.84175 & -1.77032 & -1.71643 & -1.69600 & -1.68199 & -1.67344 & -1.66946 & -1.64503 & -2.51767 \\
\hline
\end{tabular}




\section{Online Journal of Applied Knowledge Management}

A Publication of the International Institute for Applied Knowledge Management

\begin{tabular}{|c|c|c|c|c|c|c|c|c|c|c|c|}
\hline & 10 & -1.74566 & -1.57833 & -1.52725 & -1.48410 & -1.45134 & -1.43131 & -1.41712 & -1.42976 & -1.41442 & -2.26762 \\
\hline & 11 & -1.56613 & -1.41095 & -1.33713 & -1.29482 & -1.26422 & -1.25504 & -1.25081 & -1.24243 & -1.24181 & -2.08021 \\
\hline & 12 & -1.41868 & -1.25512 & -1.18112 & -1.15030 & -1.11787 & -1.10619 & -1.10305 & -1.09152 & -1.08536 & -1.94964 \\
\hline & 12 & -1.30879 & -1.14506 & -1.07954 & -1.02835 & -1.00474 & -0.99561 & -0.99906 & -0.98222 & -0.97884 & -1.83738 \\
\hline & 14 & -1.21613 & -1.05424 & -0.97708 & -0.94393 & -0.92529 & -0.90274 & -0.90242 & -0.88975 & -0.88324 & -1.74155 \\
\hline & 15 & -1.14293 & -0.97443 & -0.89981 & -0.86911 & -0.84123 & -0.82803 & -0.82369 & -0.81453 & -0.80841 & -1.66541 \\
\hline & 16 & -1.08079 & -0.91074 & -0.84248 & -0.80253 & -0.78233 & $\begin{array}{l}-0.76701 \\
\end{array}$ & -0.75767 & -0.75320 & -0.74158 & -1.60507 \\
\hline & 17 & -1.02132 & -0.86381 & -0.78788 & -0.75001 & -0.72955 & -0.71192 & -0.70148 & -0.69456 & -0.68889 & -1.54507 \\
\hline & 18 & -0.97998 & -0.81532 & -0.74306 & -0.70138 & -0.67693 & -0.66671 & -0.65126 & -0.65299 & -0.64178 & -1.49420 \\
\hline & 19 & -0.93826 & -0.77609 & -0.69819 & -0.66255 & -0.63594 & -0.62739 & -0.61698 & -0.61142 & -0.59773 & -1.45309 \\
\hline & 20 & -0.89926 & -0.73920 & -0.66217 & -0.62691 & -0.60777 & -0.58838 & -0.57722 & -0.57271 & -0.56819 & -1.41957 \\
\hline $\mathrm{p}=5$ & 6 & -7.32655 & -7.03751 & -6.98826 & -6.96635 & -6.84530 & -6.90930 & -6.87380 & -6.82420 & -6.83466 & -8.04640 \\
\hline & 7 & -4.66260 & -4.40552 & -4.29456 & -4.22599 & -4.18179 & -4.20273 & -4.19379 & -4.17371 & -4.14342 & -5.39319 \\
\hline & 8 & -3.57955 & -3.36198 & -3.24123 & -3.22007 & -3.15842 & -3.14364 & -3.13096 & -3.09061 & -3.11512 & -4.32076 \\
\hline & 9 & -2.96916 & -2.76054 & -2.61021 & -2.57630 & -2.54314 & -2.54470 & -2.50738 & -2.49888 & -2.49459 & -3.71209 \\
\hline & 10 & -2.57981 & -2.34198 & -2.23540 & -2.19179 & -2.14361 & -2.12102 & -2.09845 & -2.10045 & -2.09765 & -3.29049 \\
\hline & 11 & -2.28940 & -2.04756 & -1.94055 & -1.89305 & -1.84635 & -1.83017 & -1.82036 & -1.80443 & -1.78813 & -3.01898 \\
\hline & 12 & -2.06016 & -1.82971 & -1.71300 & -1.67337 & -1.63257 & -1.61860 & -1.59511 & -1.58219 & -1.56916 & -2.79180 \\
\hline & 12 & -1.88776 & -1.65447 & -1.55254 & -1.50808 & -1.45304 & -1.44322 & -1.42630 & -1.40895 & -1.40772 & -2.61391 \\
\hline & 14 & -1.75700 & -1.52191 & -1.42081 & -1.36102 & -1.32520 & -1.30669 & -1.28185 & -1.28558 & -1.27410 & -2.48348 \\
\hline & 15 & -1.64685 & -1.41069 & -1.30383 & -1.24456 & -1.20842 & -1.18809 & -1.16981 & -1.15558 & -1.16715 & -2.37831 \\
\hline & 16 & -1.56074 & -1.31599 & -1.20859 & -1.15332 & -1.11842 & -1.09890 & -1.08140 & -1.07708 & -1.06268 & -2.27253 \\
\hline & 17 & -1.47253 & -1.23483 & -1.12754 & -1.07686 & -1.03327 & -1.02062 & -0.99893 & -0.99111 & -0.98150 & -2.19058 \\
\hline & 18 & -1.40725 & -1.16718 & -1.06527 & -1.00055 & -0.96530 & -0.94967 & -0.93027 & -0.91958 & -0.91194 & -2.12870 \\
\hline & 19 & -1.33720 & -1.11129 & -0.99626 & -0.94371 & -0.91111 & -0.88471 & -0.87584 & -0.86023 & -0.85551 & -2.07429 \\
\hline & 20 & -1.28874 & -1.06000 & -0.95063 & -0.89690 & -0.85488 & -0.83891 & -0.82475 & -0.81308 & -0.80276 & -2.01652 \\
\hline
\end{tabular}

